\title{
A Cross-sectional Histo-morphological Study of Vesiculobullous Lesions of Skin
}

\author{
Tiwari A.K. ${ }^{1}$, Sharma $S^{2}$ Mathur D.K. ${ }^{3}$ \\ ${ }^{1}$ Dr Amit Kr Tiwari, Consultant dermatologist, Govt RBM Hospital, Bharatpur, Rajasthan, ${ }^{2}$ Dr Swati Sharma, Final year \\ Resident, Department of Pathology, MP Shah Medical College, Jamnagar, Gujrat, ${ }^{3}$ Dr Deepak K. Mathur, Sr. Professor \\ and Unit Head, Department of Skin, Vd and Leprosy, SMS Medical College, Jaipur, Rajasthan
}

Address for Correspondence: Dr Amit Kr Tiwari, Email: inbiosci@gmail.com

\begin{abstract}
Histopathology study of skin biopsies is one of the useful techniques in the investigation of vesiculobullous lesions. Out of the 70 cases of vesicobullous studied, pemphigus vulgaris was the most common type with 44 cases (61.4\%) which is followed by 25 cases $(35.7 \%)$ of pemphigus foliaceus and least percent was found with 1 cases $(2.9 \%)$ for pemphigus vegetans. Case study showed that disease was predominately observed in male in the ratio of 1.4:1 (25 males and 18 females). It was observed in Pemphigus vulgaris cases that initial lesions involves mucous membranes in $37.2 \%$ cases and $13.9 \%$ cases involves lesions including both skin and mucosae in $13.9 \%$ cases. Mucosal involvement at one time or the other was seen in 31 patients $(72.1 \%)$. In despite of this, in pemphigus foliaceus, only $4 \%$ cases showed initial mucosal involvement and involvement of both skin and mucous membrane was observed in 12\% cases. This establishes that along with clinical correlation, histo-morphological study is also a important tool in the diagnosis of Vesiculobullous skin disorders.
\end{abstract}

Keywords: Histopathology, Vesicobullous lesions, Pemphigus, Diagnosis, Skin disorders, Mucosae.

\section{Introduction}

Vesiculobullous disorders represent a heterogenous group of dermatoses with protean manifestations. They have severe economic consequences as well have dramatic impact on the family and health services. These skin diseases have been the subject of broad investigation in recent years [1]. Wide variety of bullous diseases are there, some of which can be very fatal, some bullous lesions may have serious sequele, and required early treatment and intervention to lesser the further morbidity and mortality [2]. Clinical examination of skin bullous lesion provides dermatologist gross morphological finding upon which differential diagnosis can be found out. However histopathological examination is needed for definite diagnosis [3]. A major source of dismay to pathologist is Bullous lesions. Skin biopsie are easily deliberate with precision, direct immunofluorescent microscopy in conjugation with histopathology and it gives the best diagnostic yield in bullous lesions and also make a clear reporting [4].

\section{Case Presentation}

This study was done at SMS Medical College, Jaipur (Rajasthan). A detailed history with particular reference to the mode of onset, characteristics and distribution of the lesion was taken. Punch biopsy of early vesiculo-bullous lesions were carried out in patients with biopsy punch of $4 \mathrm{~mm}$ or $5 \mathrm{~mm}$ diameter. The selection of the site for the biopsy is very critical step and lesion should be selected carefully. Histopathological examination may be misleading if lesions is not fully developed and uncomplicated. Lesions as well as normal surrounding tissue both are included in biopsy specimen, so that advancing border of the lesion can be visualized for histopathological examination of vesicles and bullae. It is

Manuscript received: $02^{\text {nd }} \mathrm{Feb} 2016$

Reviewed: $12^{\text {th }}$ Feb 2016

Author Corrected: $22^{\text {nd }}$ Feb 2016

Accepted for Publication: 03 ${ }^{\text {rd }}$ March 2016 
important that the entire lesion be removed intact to permit the study of location of vesicle, the nature of its roof and floor and type, condition and cells present in the blister. The entire specimen should be fixed in formalin and embedded in paraffin [5]. Cytological smear (Tzank smear ) were carried out where it is possible because most of the patient come with older lesions with crusting so it was not possible to take smear in all patients. To make Tzank smear, intact roof of a blister is opened along one side, folded back and the floor is gently scraped. The material thus obtained is smeared on microscopic slide, air dried, and stained with Haematoxylin \& Eosin [6]. A vast array of under lying pathologies derive the vesiculobullous lesions, the spectrum of disease encountered ranged from inherited disorders to acquired disease liked drug reactions. Bullous pemphigoid, occurring as multiple, tense bullae of varying sizes commonly in adults, had the highest incidence, followed by pemphigus vulgaris, in which oral lesions were predominant with skin involvement showing flaccid bulla of varying sizes, erythema multiforme, presenting as popular erythematous eruptions caused by a variety of unrelated stimuli, dermatitis herpetiformis, which occurred along with gluten sensitive enteropathy with skin involvement showing vesicles on erythematous bases and pemphigus foliaceus, which had characteristic positive Nikolsky's sign and a few uncommon conditions like superficial pustular dermatoses and Darier's disease were also encountered. Out of the 70 cases of vesicobullous studied, pemphigus vulgaris was the most common type with 44 cases $(61.4 \%)$ which is followed by 25 cases $(35.7 \%)$ of pemphigus foliaceus and least percent was found with 1 case $(2.9 \%)$ for pemphigus vegetans.

Male was predominately afflicted with this disease in the ratio of 1.4:1 (25 males and 18 females). Out of the 44 cases of pemphigus vulgaris, 37 patients (88.4\%) were of age between 21 and 60 years; and 1 case (4.6\%) was below 20 years of age. Only 2 cases $(6.9 \%)$ were above 60 years of age. Out of the 25 cases of pemphigus foliaceus, 20 patients $(80 \%)$ were lying in the age group of 21-60 years; while 2 patients (8\%) were older than 60 years and 3 patients (12\%) were below 20 years. One case of pemphigus vegetans was seen of the age 25 years. It was observed in Pemphigus vulgaris cases that initial lesions involves mucous membranes in 37.2\% cases and $13.9 \%$ cases involves lesions both in skin as well as in mucosae. 31 patients were seen to be having mucosal involvement at one time or the other was seen in 31 patients (72.1 $\%)$. In despite of this, in pemphigus foliaceus, only $4 \%$ cases showed initial mucosal involvement while in $12 \%$ cases involvement of both skin and mucous membrane was seen.

The nature of the lesions observed during the course of pemphigus is shown in (Table 1). All cases were found to be suffering from flaccid bullae. Blisters seen arising on nonerythematous skin was seen in 42 cases (95.45\%) of pemphigus vulgaris, which spontaneously ruptured to give rise to erosions in 23 cases $(52.2 \%)$. Crusted lesions, erythematous plaques, vegetations and pustules were present less frequently. Skin lesions were observed on common sites in pemphigus vulgaris were generalised involved in 16 cases $(37.2 \%)$, on face in 9 Cases $(20.9 \%)$, scalp in 16 cases $(18.6 \%)$. On the other hand, in pemphigus foliaceus, lesions were generalised distribute in 8 cases $(32 \%)$, on face in 7 cases $(28 \%)$, on scalp in 4 cases $(16 \%)$, on trunk in 4 cases $(56 \%)$ and involvement of extremities in 12 cases (48\%). In pemphigus foliaceus, blisters arising on erythematous skin were seen in 17 cases $(68 \%)$, crusted lesions in 10 cases (40\%), erosions in 11 cases (44\%) and pustules in $8 \%$ cases. Vegetations were seen in a single case of pemphigus vegetans. Pruritus was seen in 14 cases $(20 \%)$ of all pemphigus cases. Eleven cases (25.6\%) of pemphigus vulgaris complained of pruritus. As compared to a solitary case (4\%) of pemphigus foliceus, single case (100\%) of pemphigus vegetanshadpruritus. Out of 36 cases of pemphigus vulgaris and 19 cases of pemphigus foliaceus with the history of Nikolsky's sign , it was positive in 35 cases $(97.2 \%)$ and 18 cases $(94.7 \%)$ respectively. A case of pemphigus vegetans showed positive Nikolsky's sign.

Of the 44 cases of pemphigus vulgaris, 32 cases (72.7\%) showed intra-epidermal suprabasal vesicles and 11 cases (25.0\%) showed mid-epidermal vesicles (Table 2). Acantholysis affecting follicular sheath was seen in $40.9 \%$ cases. An inflammatory infiltrate was present in the bulla cavity in 25 cases $(56.8 \%)$. Neutrophils were predominant in 9 cases (20.9\%) and eosinophils 11 cases (25.6\%). Midepidermal vesicles which may be due to regeneration of the cells from the floor of the bulla were seen in old bullae. Acantholysis, as groups of cells or single cells within the bulla cavity, was seen in $79.5 \%$ cases. Dyskeratosis, basal layer budding and pseudo-epitheliomatous proliferation was not seen in any of the cases. Eosinophilic spongiosis was seen in 1 case $(2.2 \%)$. This was only known case of pemphigus and had developed new lesions after discontinuation of steroids. In 11 cases $(17.6 \%)$, the epidermis was lost during the process. Previously negative report was observed in 5 of these cases (11.6\%) and could be diagnosed on the basis of the above histological features. 
Table-1: Nature of lesions observed during the study

\begin{tabular}{|l|l|l|l|l|}
\hline S.No. & Type of Pemphigus & Pemphigus vulgaris & Pemphigus foliaceous & Pemphigus vegetans \\
\hline 1. & Vesicle /Bulla & & & \\
& $\bullet$ & $44(100 \%)$ & $25(100 \%)$ & $1(100 \%)$ \\
& $\bullet$ & - & - & - \\
& $\bullet$ & $02(4.5 \%)$ & $08(32 \%)$ & - \\
& $\bullet$ Elaccid & $42(95.45 \%)$ & $17(68 \%)$ & $01(100 \%)$ \\
\hline 2. & Erosionse & $23(52.27 \%)$ & $11(44 \%)$ & - \\
\hline 3. & Crusted lesions & $8(18.18)$ & $10(40 \%)$ & - \\
\hline 4. & Vegetative lesions & $8(18.18)$ & $2(8 \%)$ & $01(100 \%)$ \\
\hline 5. & Erythematous plaques & $3(06.8 \%)$ & - & - \\
\hline 6. & Pustules & $2(4.5 \%)$ & $2(8 \%)$ & - \\
\hline
\end{tabular}

Table-2: Histopathological changes in Pemphigus vulgaris

\begin{tabular}{|l|l|l|l|}
\hline S.No. & Histopathological feature & No. of cases & Percentage \\
\hline 1. & Suprabasal clefts/bulla & 32 & 72.72 \\
\hline 2. & Mid- epidermal vesicle & 11 & 25.00 \\
\hline 3. & Acantholysis & 35 & 79.54 \\
\hline 4. & Row of Tombstone appearance & 23 & 52.27 \\
\hline 5. & Eosinophilic spongiosis & 01 & 02.27 \\
\hline 6. & Infiltrate in bulla cavity & 25 & 56.81 \\
\hline 7. & Dyskeratosis & - & - \\
\hline 8. & Basal layer budding & - & - \\
\hline 9. & Acantholysis involving follicular sheath & 18 & 40.90 \\
\hline
\end{tabular}

Table-3: Histopathological changes in Pemphigus Foliaceous

\begin{tabular}{|l|l|l|l|}
\hline S. No. & Histopathological features & No. of cases & Percentage \\
\hline 1. & Sub-corneal bulla & 13 & 52 \\
\hline 2. & Sub-granular bulla & 08 & 32 \\
\hline 3. & Mid-epidermal bulla & 02 & 08 \\
\hline 4. & Dyskeratosis & 04 & 16 \\
\hline 5. & Hyperkeratosis & 10 & 40 \\
\hline 6. & Parakeratosis & 12 & 48 \\
\hline 7. & Acanthosis & 07 & 28 \\
\hline 8. & Papillomatosis & 05 & 20 \\
\hline 9. & Acantholysis & 24 & 96 \\
\hline
\end{tabular}

In case of pemphigus vegetans hyperkeratosis, papillomatosis and irregular acanthosis with the development of intraepidermal eosinophilic abscesses was observed. Suprabasal lacunae with a few acantholytic cells were observed. This case was of Neumann type. Pemphigus erythematosus, drug-induced pemphigus and paraneoplastic pemphigus was not seen in this case.

In case of pemphigus foliaceous Dyskeratosis, pseudoepitheliomatous proliferation and basal layer budding were not 
seen in any of the cases (Table 3). Among the 25 cases of pemphigus foliaceus studied, acantholysis was observed in 24 cases $(96 \%)$. In $46.5 \%$ cases, acantholysis affecting follicular sheath was observed. Subcorneal bulla was seen in 13 cases $(52 \%)$, subgranular cleavage from middle epidermis in 08 cases $(32 \%)$. An inflammatory infiltrate was present in the bulla cavity in 23 cases (53.5\%). In 3 cases eosinophilic spongiosis was seen (6.9\%). Two of them were known cases of pemphigus and it was observed that discontinuation of steriods were followed by development of new lesions . In 8 cases (18.6\%), the epidermis was lost during the process. In 9 cases (20.9\%) neutrophils were predominant and eosinophils in 11 cases $(25.6 \%)$. There was negative report was observed in five of these cases $(11.6 \%)$ and could be diagnosed on the basis of the above histological features.



Fig 1: Acantholytic cells seen in blister Fluid

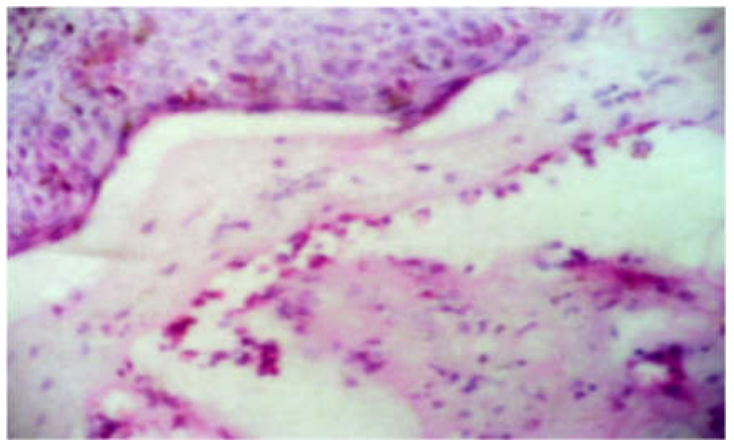

Fig 2: Subepidermal bulla with eosinophils

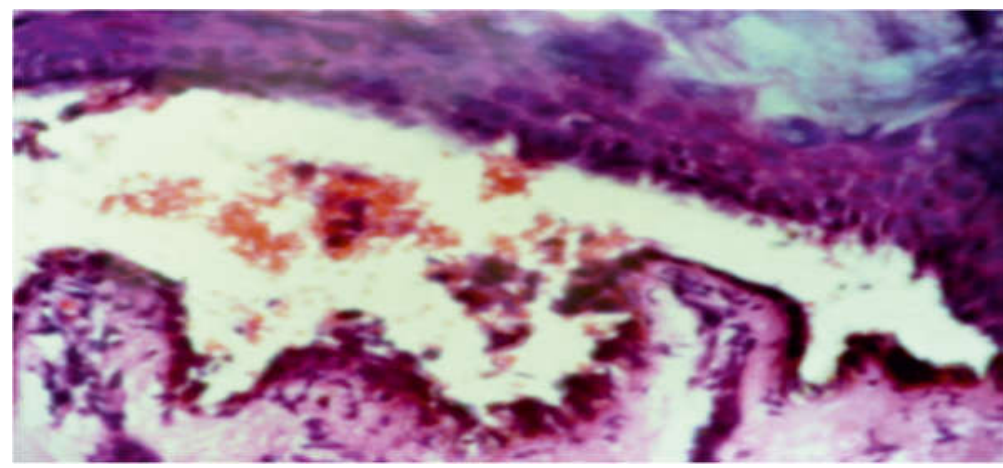

Fig 3: Suprabasal bulla with acantholytic cells

Out of 25 case of pemphigus foliaceus, in 10 cases (83.3\%) the infiltrate comprised of polymorphs. Spongiosis was seen in 10 cases $(40 \%)$ and exocytosis in 8 cases (32\%). In a few cases, acanthosis, hyperkeratosis, parakeratosis, papillomatosis and increased pigment formation were also seen. 24 cases (96\%) showed acantholysis. Subcorneal bulla was seen in 13 cases (52\%), 8 cases were observed to be subgranular cleavage from middle epidermis (32\%). Dyskeratosis was seen in only 4 cases (16\%).In 12 cases, inflammatory cells were seen in the bulla cavity (48\%).

\section{Discussion}

Among the Vesiculobullous, in the pemphigus group, pemphigus vulgaris is the commonest variety [7]. There was 109 cases of pemphigus from Tripoli, libya have observed by Shafi, $M$ et al among that the incidence of pemphigus in Libya is very high, with predominant variant being pemphigys foliaceus[8]. In his study Shafi. $M$ et al also found out that males were predominantly affected than females [8]. In the present study, most of the cases of Pemphigus vulgaris were observed followed by pemphigus foliaceus. A single case of pemphigus vegetans was noticed. Fabbri P et al stated that acantholysis was the major mechanism involved in pemphigus vulgaris and its variant pemphigus vegetans there was suprabasilar clefting, which was the hallmark of these diseases [9]. In pemphigus foliaceus and its variant pemphigus erythematosus, the pathologic alteration of the epidermis occurs superficially i.e in the granular layer $[9,10]$. The pemphigus group was found to have same observations in the present study, with all the cases of pemphigus group showing acantholysis as the predominant mechanism of bulla formation. It has been 
poorly understood the reason for the intrinsic difference between pemphigus foliaceus and pemphigu vulgaris , but has long been of interest to clinicians as well as the investigators [11].

Funding: Nil, Conflict of interest: None. Permission of IRB: Yes

\section{References}

1. Wojnarowska F, Venning VA. Immunobullous diseases. In: Burns T, Brethnach S, Cox N, Griffiths C, ed. Rook's Textbook of Dermatology, 8th edition. Oxford: Wells Blackwell, 2010, 40, 1-40.62.

2. Tani M, Shimizu R, Ban M, Murata Y, Tamaki A. Systemic lupus erythematosus with vesiculobullous lesions. Immunoelectron microscopic studies. Arch Dermatol. 1984 Nov;120(11):1497-501.

3. Kabir AN. Direct immunofluorescence test of skin biopsy samples results of 204 cases. Dinajpur Medical College Journal. 2009; 2(1): 8-21

4.Minz RW, Chhabra S, Singh S, Radotra BD, Kumar B. Direct immunofluorescence of skin biopsy: perspective of an immunopathologist. Indian J Dermatol
Venereol Leprol. 2010 Mar-Apr;76(2):150-7. doi: 10.4103/0378-6323.60561.

5. Zuber TJ. Punch biopsy of the skin. Am Fam Physician. 2002 Mar 15;65(6):1155-8, 1161-2, 1164.

6.Gupta LK, Singhi MK. Tzanck smear: a useful diagnostic tool. Indian J Dermatol Venereol Leprol. 2005 Jul-Aug;71(4):295-9.

7. Korman N. Pemphigus. J Am Acad Dermatol. 1988 Jun;18(6):1219-38.

8. Shafi M, Khatri ML, Mashina ML et al. Pemphigus: A clinical study of 109 cases from Tripoli; Libya. Indian journal of Dermatology Vernerology and Leprology. 1994; 60:140-143.

9. Fabbri P, Lotti T, Panconesi E . Pathogenesis of pemphigus the role of epidermal plasminogen activators in acantholysis. International Journal of Dermatology. 1985; 422-425.

10.Bean SF, Lynch FW. Senear-Usher syndrome (pemphigus erythematosus). Immunofluorescent studies in a patient. Arch Dermatol. 1970 Jun;101(6):642-5.

11.Lynch PJ, Gallego RE, Saied NK. Pemphigus -- a review. Ariz Med. 1976 Dec;33(12):1030-7.

\section{How to cite this article?}

Tiwari A.K., Sharma S, Mathur D.K. A Cross-sectional Histo-morphological Study of Vesiculobullous Lesions of Skin. Int J Med Res Rev 2016;4(3):392-396. doi: 10.17511/ijmrr.2016.i03.19 